\title{
EFFICIENT ROUTING IN AD HOC NETWORKS WITH DIRECTIONAL ANTENNAS
}

\author{
David L Rhodes \\ OpCoast \\ Brick, NJ
}

\begin{abstract}
Increased mobility, connectivity and self-formation are the hallmarks of envisioned tactical communication systems. Tactical military systems, with little or no fixed infrastructure and critical QoS demands, represent one of the biggest challenges in the area of ad hoc networks. These systems must operate in varied and challenging propagation environments possibly with adversarial jamming or intrusion attempts. The use of nodes with directional antennas is advocated to solve some of these problems. However, generalized routing for networks with directional antenna equipped nodes has had little attention. A new method for determining routes by deciding network-optimized directional antenna settings is presented.
\end{abstract}

\section{INTRODUCTION AND BACKGROUND}

The tactical communication networks envisioned for the Future Force and Joint Vision 2020 stand among the most challenging operating regimes for wireless networks. In addition to having no (or little) fixed infrastructure, such networks must operate with high levels of security in challenging environments ranging from subterranean spaces and caves to long-haul foliaged terrain to indoors in modern buildings with node mobility rates ranging from walking to sub-sonic speeds. Communication services should be maintained at maximum operating levels even when adversarial jamming or intrusion is attempted. Several recent sub-scale experiments and simulations have clearly exhibited the challenge, showing that inter-radio link connectivity may rapidly change and the difficulties of maintaining or determining routes in what might be viewed as a network with a 'chaotic' topology.

While unicast routing over the large-scale, wired Internet has largely settled on OSPF (open shortest path first), RIP (routing information protocol) and BGP (border gateway protocol) [2], routing in mobile ad hoc networks remains a subject of research. Some of the primary techniques are AODV (ad hoc on-demand distance vector) [3], ZRP (zone routing protocol) [4], TORA (temporally-ordered routing algorithm) [5], DSR (dynamic source routing) [6], DSDV (destination-sequenced distance-vector) [7] [23], and OLSR (Optimized Link State Routing Protocol [12]. OLSR and DSDV attempt to actively maintain routing tables, while the other methods are 'on demand' meaning that they determine routes on an 'as needed basis.' OLSR, AODV and TORA require links that are symmetric, while methods such as DSR and ZRP do not require this assumption. Link asymmetry may be caused in several ways: when transmit power adjustments are used; for packetswitched directional antennas; for a different noise or jamming environment at each end of the link; or for compatible but heterogeneous radios. Some research [8] shows that asymmetry may be common in ad hoc networks. A summary and performance comparisons of several unicast ad hoc routing protocols can be found in [8] and [9].

Several multicast methods have been proposed for ad hoc networks. Anonymous GOSSIP [10] layers on top of other multicast protocols to improve reliability (at the cost of additional overhead), MAODV (multicast AODV) [11] extends the route request/response mechanism of AODV to develop a multicast routing tree on demand (also assuming link-level symmetry), and OMDRP (on demand multicast routing protocol) [13] allow asymmetric links (and can also be used for unicast routing). Unlike MAODV, OMDRP creates a multicast mesh rather than a tree since nodes on any path from source to destinations become forwarding nodes. While this improves packet delivery especially when moderate mobility exists [14], it also significantly increases traffic during sends, routing overhead, the number of transmissions and media collisions. Although further study is warranted, it seems that this approach will scale far less efficiently, especially in strongly connected graphs. The use of 'nonuniform $m$-PSK constellations' channel coding for multicast has also been considered [15]. It focuses on the issues surrounding a single link, since a multicast group would typically be restricted to using the minimum data-rate receivable across all group members. As will be shown, non-uniform coding gives rise to links with multiple metrics in a multistate graph.

The use of directional antennas in ad hoc networks is now gaining attention in the research community. While earlier studies were mostly confined to MAC and link layer issues (e.g. [16], [17]), recent work [18] includes routing levels. The effort in [19] includes directional transmit antennas (assuming omni directional receivers) and computes a multicast tree comprised of optimizing goodput in an energy limited system. The method assumes knowledge of node locations and does not include mobility (thereby 
eliminating the acquisition problem) nor the routing protocol needed to form and maintain the tree.

Of course, the use of directional antennas is inherently cross-layer as changes in antenna states can appear as intermittent link losses or reestablishments to the routing layer. In the case where the antenna is rapidly steering, this might appear as route flapping; and deafness will result when the antenna is never pointed/adjusted to allow particular connections. Of course, mobility itself will also cause link connections and net topology to vary, the difference being that we are explicitly controlling the directional antenna(s) for the purpose of networking (assuming that the mobility is not specifically being used for network formation as might be the case for moveable ground or air nodes dedicated entirely to communication).

\section{THE PROBLEM}

Use of links that dynamically adjust data-rate to RF environmental conditions (via Tx power adjustment or nonuniform coding [15]) and directional antennas give rise to the notion that link metrics are multi-state. That is, the graph that represents the network has edge metrics that may take on different values depending on conditions or antenna state. While some work has been done on link-layer and MAC layer issues (e.g. [20]), a general network-routing level solution where nodes include directional antenna effects has not been presented until now. One somewhat related effort [21], however, does discuss how to use angle of arrival to adjust Tx power level to better form a network, but is not geared to controlling the antennas for the purpose of routing.

Since antenna states at each node are in general independent, there are an exponential number of combinatorial states in the multi edge-metric solution graph. Our new multi-state routing algorithm (called MSD-SPA) [1] is ideal for treating the exponential complexity of computing routes in such a multi-state network. This is achieved by only finding solutions for a 'dominant set' of combinations that use don't cares to correctly represent all state combinations and by leveraging dynamic programming to further lower computational cost. We focus the paper on the topic of directional antennas, but the core algorithm has applicability to any case where multiple edge metrics arise, such as rate-adaptable RF links.

Figure 1 depicts an antenna gain pattern for node $v_{1}$ (indicated as a star rather than round shaped node) that may take on any of four states (called 'State 1' through 'State 4 '), the gain is meant to be larger in the direction of larger radius (e.g. in 'State 4' the largest gain is in the direction of node $v_{3}$ ). Due to link adaptation, the achievable datarate, and even basic connectivity, between any pair of nodes may depend on the antenna state.

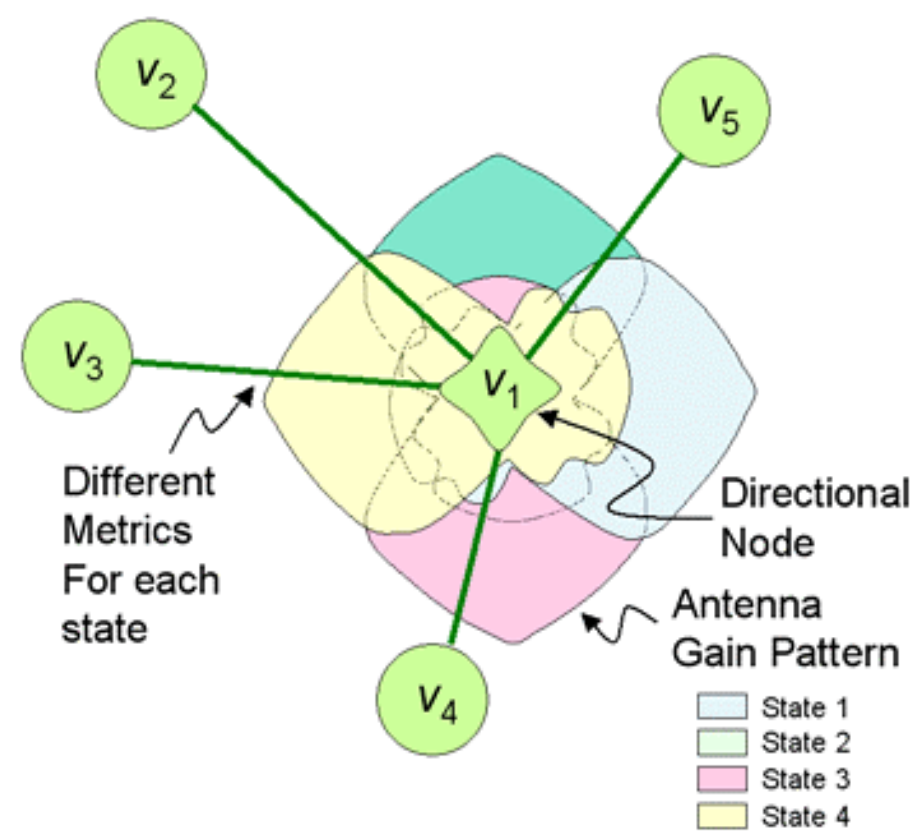

Fig. 1. A sample 4-state directional antenna utilized by node $v_{1}$ gives rise to multiple metric states to other nodes (with omni-antenna).

Figure 2 shows a possible graph resulting from a radio system composed of nodes with directional antenna (ignore the dashed lines for the moment). In the figure, there are nine radio nodes, four of which are directional $\left(v_{1}, v_{4}, v_{5}\right.$ and $V_{9}$ ). If each of the four directional nodes has four directional states, then there are $4^{4}=256$ possible overall combinations of directional states. However, as only pairwise combinations must be considered and not the complete combinatorial space, if $m$ is the number of antenna states and $n$ is the number of nodes with directional antennas, then there are

$$
m^{2}\left(n^{2}-n\right)
$$

combinations (192 in our sample). Based on receiver sensitivity, propagation and related effects, these states will give rise to various inter-node connectivity, which are then mapped to a multi-state graph problem as notionally shown in the figure. It is important to realize that there are two different 'states' being discussed, the first is the state of directional antenna at each node and the second is the resulting multi-metric edge state graph that results from analyzing the antenna states and translating them into the multistate graph (e.g. Fig 2). As will be discussed next, multistate edge metrics in the solution graph are derived on a pairwise analysis basis from the antenna states.

In the case of Figure 2, edge connectivities are shown as either ' 1 ' or ' $1, \infty$ ' meaning that this edge may be connected or disconnected based on antenna state, possible 


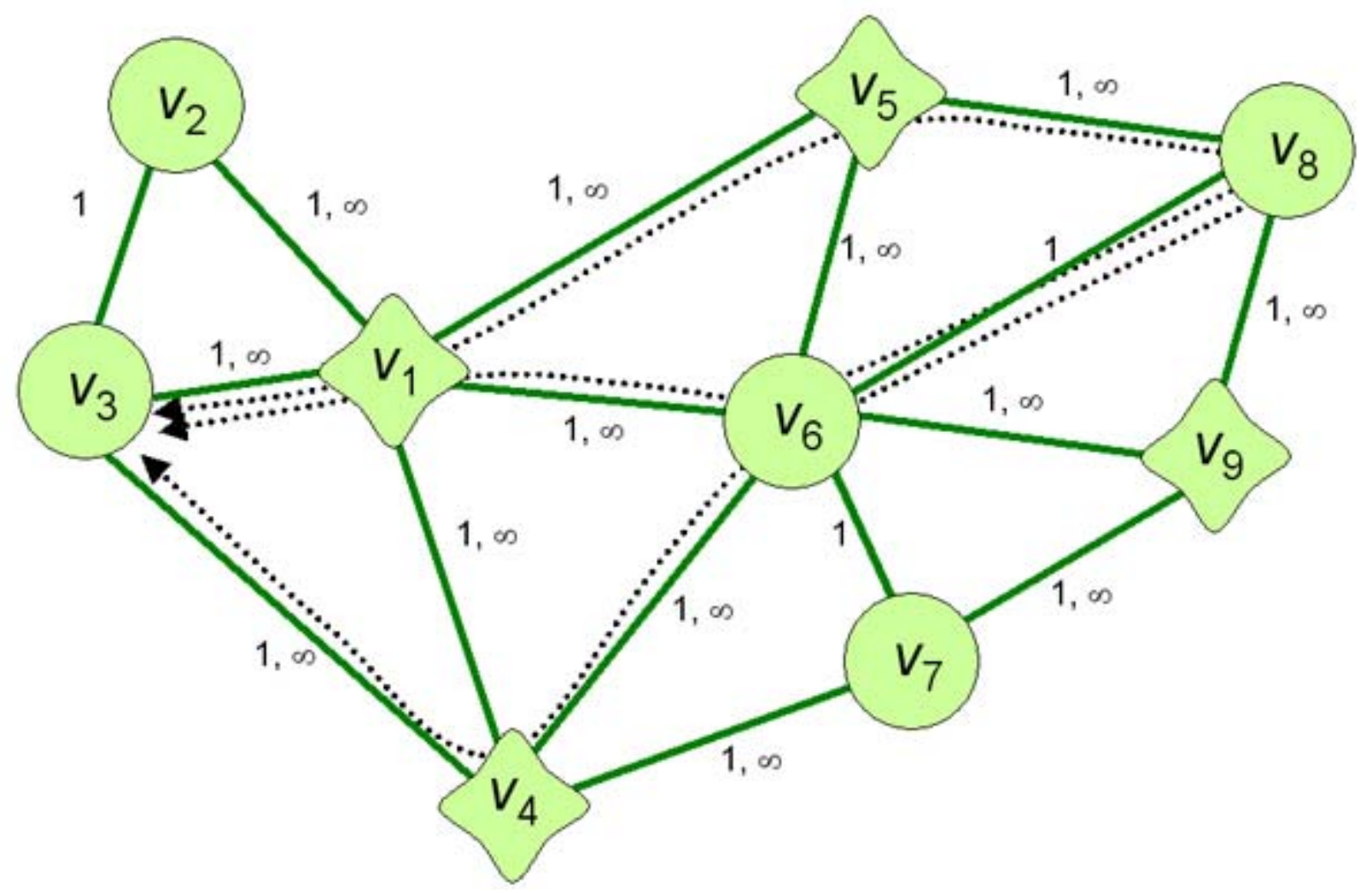

Fig. 2. Simple multi-metric edge arising from nodes with directional antenna.

mutual antenna states (e.g. pairwise connectivity may occur only if both antenna are pointing in a certain direction). The edges in Figure 2 are shown as un-directed, but each of these is meant to imply a directional connection that has the same metrics in both directions ${ }^{1}$.

In Figure 2, there are thirteen arcs shown (representative of the 26 directed arcs in the actual graph) each with two states giving rise to $2^{26}=67,108,864$ combinatorial states in the multistate graph. Assuming that the root of the multi-state tree (source node) is $v_{8}$, the MSD-SPA algorithm can determine all shortest path distances to all vertices with an associated tree for all possible state combinations (e.g. all $67+$ million) by analyzing only 836 cases $^{2}$. In addition to the savings of over four orders of magnitude (in this case), it also leverages dynamic programming techniques; in this case the average size of the sub-solution copied to new solutions is about $71.9 \%$ giving rise to almost another factor of four savings over a brute force approach (which would be untenable). The method is also flexible enough to permit solution pruning to provide

1 Assuming symmetry in propagation and $\mathrm{Tx} / \mathrm{Rx}$ properties. Otherwise the edges in each direction have different metrics and this is fully allowed here.

2 Shortest path distances are unique for any edge metric state setting, but there may be multiple trees/paths that provide these distances. The MSD-SPA method can be altered to find all equivalent trees if desired. guaranteed polynomial time execution at the expense of not exploring all possible settings (e.g. limiting the search in various ways). The core algorithm is described next, along with how it is applied in the case of routing from $v_{8}$ to $v_{3}$ as a sample.

\section{THE MSD-SPA ALGORITHM}

The new core algorithm for treating multistate networks, called the multistate dynamic shortest path algorithm (MSD-SPA), is flow charted in Figure 3. Algorithmic details including correctness proofs are in [1] so only a short summary of the method is presented here.

A graph-state is a graph with all edges set to particular edge metric values (where shortest paths might be typically found with Dijkstra's method). The concept of dominant-state is used in MSD-SPA and broadens the definition of graph-state to also allow 'don't care' settings for some of the edge metrics. It is defined with respect to a starting vertex $s$ :

\section{Definition: Dominant-State}

A particular setting of edge metrics, including don't care settings, is called dominant if and only if altering any edge metric setting(s) will change the shortest reachable distance from $\mathrm{s}$ to some vertex and where the state is not in turn dominated by another dominant state. 
Finally, the dominant-set provides a solution to the multistate shortest path problem:

\section{Definition: Dominant Set}

The dominant-set of dominant-states is the set of dominant-states such that the associated graph is 'covered" meaning that any possible graph state can be matched to a member in the dominant-set. By the definition for dominant-state none of the states in the dominant set dominate any other.
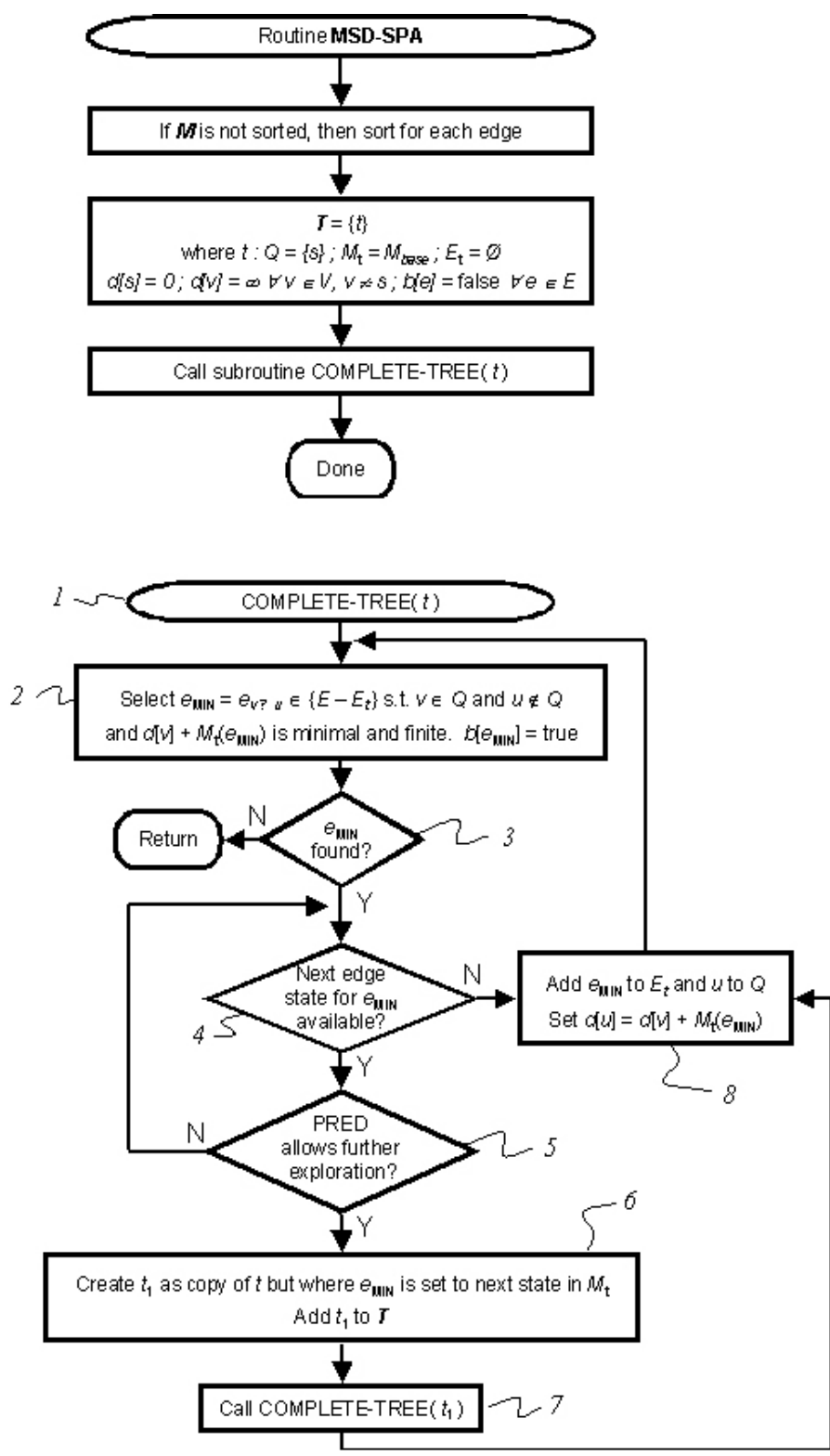

Fig. 3. MSD-SPA

The problem is to find multiple shortest path trees from a given source vertex to all other vertices for all possible states of the graph. This problem is a variation of the well- known single-source shortest path problem, with the difference being that edge metrics are not fixed values but are allowed to take on multiple possible choices. So the problem becomes finding the shortest path trees from a source vertex $s$ for all combinations of edge metric values.

As alluded to before, the MSD-SPA algorithm efficiently finds the dominant-set solution in multi-state graphs but proofs are beyond the scope of this paper. While the method is exponential in the worst case, provisions for limiting complexity at the possible cost of lack of optimality is provided. This is controlled by limiting recursive exploration (the 'PRED' function at flow chart step 5 in Figure 3). See [1] for complete exposition and algorithmic proofs for MSD-SPA.

Benefits of the approach are manifold. By efficiently analyzing all possible edge metric combinations, including disconnects (an edge metric of infinity), the method provides:

- Ability to find optimal routing trees/paths even in large networks with many combinatorial states

- Pre-computed alternate routes for degraded or enhanced network states (e.g. when one or more links fail or is able to receive at higher data-rates). This is can enhance an unicast routing method such as DSR (which uses source directed routing) and other unicast routing methods as it will enable virtually instantaneous switch over to an alternate paths due to the availability of multiple pre-computation routes.

- Ability to consistently treat both directional antennas and variable data-rate links. Note that the preferential reception may be from use of nonuniform $m$-PSK constellations or other automated link adaptation techniques (e.g. 802.11's data-rate adaptation based on connection quality).

- Provides a way to include any combination of either omni- or directional antenna on either transmit or receive nodes. Note that we assumed both directional receivers and transmitters in the sample (Fig. 2) translation provided, but this is optional.

Note the list above includes the topic of variable data-rate links, which will be discussed in more depth later.

\section{FINDING NETWORK ROUTES}

In the case of the sample in Figure 2, assuming that node $v_{8}$ is the source and $v_{3}$ is the destination, the MSD-SPA method will find the shortest path routes of length 3 for various edge settings as shown by the dotted lines. The dominant-state entries corresponding to these solutions in the overall dominant-set solution found will define how the metrics for each edge must be set in order to achieve 
these shortest paths ${ }^{3}$. These edge metrics can then be reverse-mapped to antenna settings (the 192 cases) for routing purposes. It is assumed that the antenna can be (electronically) switched on a per-packet basis to achieve the desire routing.

A summary overview of the steps is:

1. enumerate pairwise directional antenna states into a multistate graph; in our sample graph, there are 192 (from Eq. [1]) pairwise directional antenna states.

2. efficiently solve the resulting multistate graph for all possible state combinations using the MSD-SPA method;

3. decide on routes by selecting appropriate solutions from the dominant-set. Note that this might be for routes to multiple destinations (and multicast solutions); and

4. reverse map routing decisions of step 3 to network state to define directional controls for each connection.

Although the complete data for the sample (Fig 2) case is too voluminous, we illustrate some of the tables and show how antenna control directions can be derived from the steps above. For step 1, Table I shows how to derive edge metrics from pairwise antenna states, in this case for Nodes 6 and 1 using a compass point basis for simplicity (although the states can be anything).

\begin{tabular}{|c|c|c|c|c|c|c|c|c|}
\hline \multicolumn{4}{|c|}{ Node 6, State } & \multicolumn{4}{|c|}{ Node 1, State } & \multirow{2}{*}{$\begin{array}{c}\text { Metric } \\
\text { e[v6 -> v1] }\end{array}$} \\
\hline$E$ & $w$ & $N$ & $s$ & $E$ & $w$ & $N$ & $s$ & \\
\hline \multirow[t]{4}{*}{1} & & & & 1 & & & & $\infty$ \\
\hline & 1 & & & 1 & & & & 1 \\
\hline & & 1 & & 1 & & & & 1 \\
\hline & & & 1 & 1 & & & & $\infty$ \\
\hline \multirow[t]{4}{*}{1} & & & & & 1 & & & $\infty$ \\
\hline & 1 & & & & 1 & & & $\infty$ \\
\hline & & 1 & & & 1 & & & $\infty$ \\
\hline & & & 1 & & 1 & & & $\infty$ \\
\hline \multirow[t]{4}{*}{1} & & & & & & 1 & & $\infty$ \\
\hline & 1 & & & & & 1 & & $\infty$ \\
\hline & & 1 & & & & 1 & & $\infty$ \\
\hline & & & 1 & & & 1 & & $\infty$ \\
\hline \multirow[t]{4}{*}{1} & & & & & & & 1 & $\infty$ \\
\hline & 1 & & & & & & 1 & $\infty$ \\
\hline & & 1 & & & & & 1 & $\infty$ \\
\hline & & & 1 & & & & 1 & $\infty$ \\
\hline
\end{tabular}

As can be seen, there are two cases where connectivity is established when Node 6 is in state either $W$ or $N$ and Node 1 is in state $E$, otherwise no connectivity exists. Thus the multistate edge metric for $v_{6}$ to $v_{1}$ is set to $\{1, \infty\}$. This is derived for all pairwise antenna states.

In step 2 of the general procedure, the MSD-SPA method is used to find edge metric states where the minimum distance (hop count in this case as we are using 1 as a nominal edge metric) to the destination (we are still considering $v_{8}$ to $v_{3}$ as source and destination per the example). A portion of the solution is shown in Table II. This is for the path $v_{8}$ to $v_{6}$ to $v_{1}$ to $v_{3}$ and it expresses that the metrics for the given edges must be valued/state ' 1 ' to achieve this. It will also show edges such as to $v_{8}$ to $v_{9}$ as 'don't cares' for this setting.

Table II. Abbreviated Setting Entry and distance from MSD-SPA

\begin{tabular}{|c|c|c|}
\hline v1 $\mathbf{- >}$ v3 & v6 $\mathbf{- >}$ v1 & d[v3] \\
\hline \hline Metric=1 & Metric=1 & 3 \\
\hline
\end{tabular}

Note that the solution table will show multiple settings for the route $v_{8}$ to $v_{3}$ with minimum distance of 3 and in step 3 of the procedure one of these is chosen for routing (other factors can be included in this decision as well). Once a particular solution is selected, the multi-metric edge states required are reversed mapped back to antenna states in step 4. As part of the example used here, we see that a metric of 1 is required for the edge $v_{6}$ to $v_{1}$ and using data such as Table I we see that this requires antenna states for Node 6 to be in either $W$ or $N$ states and Node 1 to be in state $E$.

Although the focus of this paper is on communication systems with directional antennas, the multi-state approach is also applicable to other areas where links can take on different metrics. For example, consider a pair of radios as in Figure 4. As shown in Fig. 4a, the data-rate achievable between these radios varies from unconnected (a 0 datarate), at a rate ' $b$ ' and at a rate ' $2 b$ ' (units are arbitrary, perhaps bits/s). Here, edge metrics are reflective of datarate instead of base connectivity. Since shortest paths are generally used, it is appropriate to use inverse data-rate as an edge metric. As shown in Fig. 4b, this radio pair would be converted to the graph with edge metrics of $(1 / 2 b, 1 / b$ and $\infty$ ). Edges are placed in both directions, as the connection is bi-directional. The illustrative example of Figure 2 uses a nominal metric of ' 1 ' and $\infty$ for disconnects, but any number of edge metrics are supported by MSD-SPA. Furthermore, both multi-rate connections and directional antennas can be consistently and comprehensively treated, but this is a topic beyond the scope of this paper.

\footnotetext{
3 Note that routes to all vertices are found at once, we select out the ones we are interested from the dominant-set solution
} 


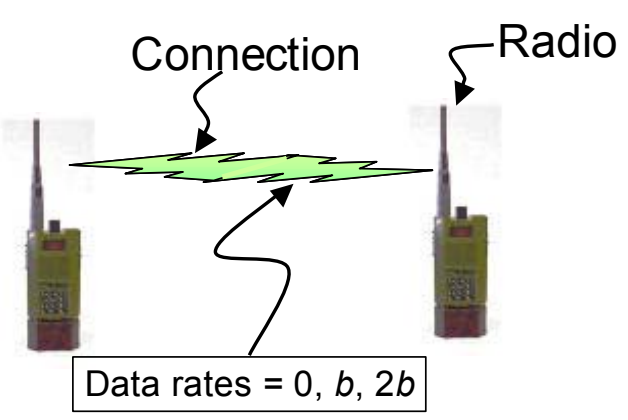

a)

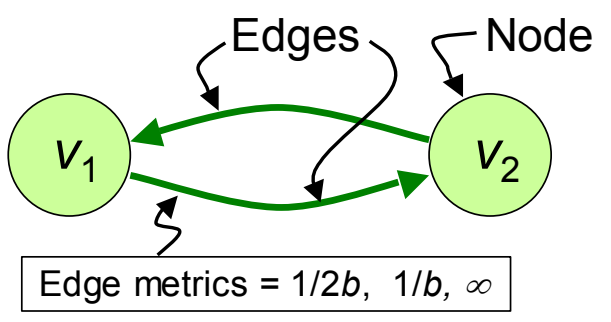

b)

Fig. 4. Multi-metric edge states may arise from radios capable of adaptable data rates. In a) two radios are capable of bi-directionally communicating at different rates and b) the graph equivalent.

\section{Protocol Issues}

Obviously, at least some changes must be made to current ad hoc routing methods to accommodate systems that utilize directional antenna-for example knowing which nodes have directional capabilities and state connectivities (i.e. like Table I) is of course a minimum requirement. Since link-state information is required by the approach, it will be simpler to combine it with ad hoc routing methods that currently provide such data such as DSDV versus a method such as AODV which only returns hop count information to routing requests, but the new method can be utilized through modifications of many mobile ad hoc routing protocols.

Another protocol issue is the exact means for directional antenna control. For example, this could be a local decision or use instructions included in the transmitted packets (like DSR). As pointed out in [23], making a series of local decisions can lead to routing loops, which implies a source directed approach might be better. Nevertheless, the issues surrounding protocol support for the method is not included in this paper. A perhaps larger but related issue is how to control 'pairwise' antenna state; obviously either a local or source directed decision can be used for determining antenna state for the current sending node, but what about a state requirement for the next hop? In much research, this is circumvented by assuming omni-directional receivers but directional transmitters. This solves the issue, and the basic approach of using MSD-SPA will still apply in such a case, but there is the opportunity loss of connectivity that directional receivers would bring.

\section{CONCLUDING REMARKS}

A novel solution to making complete routing decisions for systems equipped with directional antennas has been presented. It is capable of making decisions optimally in the face of severe combinatorial complexity, although it will be exponential in degenerate and worst-case situations, in most cases it is well within the computational abilities of current hardware. The method was illustrated in terms of unicast routing, but can also be applied to multicast routing problems as well, but this is beyond the scope of this paper.

The multi-state approach and MSD-SPA are felt to be important underlying solution components for the routing problem of directional antenna. The method has value beyond these domains as well such as for multi-state links as discussed but not fully treated here. For example, by allowing efficient pre-computation of multiple routes, it can be applied for various QoS needs and even assist in basic connectivity maintenance.

\section{REFERENCES}

[1] David L Rhodes, "Efficient Shortest Path Computation for Multistate Graphs," submitted to J. of Discrete Algorithms

[2] Sam Halabi, "Internet Routing Architectures," Cisco Press, 2000, pp. 498

[3] Charles E. Perkins, Elizabeth M. Royer. "Ad hoc OnDemand Distance Vector Routing," Proc.2nd IEEE Workshop on Mobile Computing Systems and Applications, New Orleans, LA, February 1999, pp. 90-100.

[4] Z. Haas, M. Pearlman, "ZRP: A Hybrid Framework for Routing in Ad Hoc Networks," Ad Hoc Networking, C. E. Perkins (ed), Addison-Wesley, 2000

[5] V. Park, M.S. Corson, "A highly adaptive distributed routing algorithm for mobile wireless networks," Proc. IEEE INFOCOM, April 1997, pp. 1405-13.

[6] David Johnson, David Maltz, "Dynamic Source Routing in Ad Hoc Wireless Networks," Mobile Computing (ed. T. Imielinski and H. Korth), Kluwer Academic Publishers, Vol. 353, 1996

[7] C. E. Perkins, P. Bhagwat, "Highly dynamic destination sequenced distance vector routing (DSDV) for mobile computers," Computer Communications Review, Oct. 1994, pp. 234-244.

[8] D. Ganesan, B. Krishnamachari, A. Woo, D. Culler, D. Estrin, S. Wicker, "An Empirical Study of Epidemic Algorithms in Large Scale Wireless Multihop Networks," Intel Research, IRB-TR-02-003, 14 Mar. 2002 
[9] Elizabeth Royer, C-K Toh, "A Review of Current Routing Protocols for Ad-Hoc Mobile Wireless Networks," IEEE Personal Communications Magazine, Apr. 1999, pp. 45-55

[10] Ranveer Chandra, Venugopalan Ramasubramanian, Kenneth P. Birman, "Anonymous Gossip: Improving Multicast Reliability in Mobile Ad-Hoc Networks," Proc. 21st Intl. Conf. Distributed Computing Systems (ICDCS), Phoenix, April 2001, pp. 275-283.

[11] Elizabeth M. Royer, Charles E. Perkins, "Multicast Operation of the Ad hoc On-Demand Distance Vector Routing Protocol," Proc. MobiCom, Seattle, WA, August 1999, pp. 207-218.

[12] T. Clausen, P. Jacquet, A. Laouiti, P. Muhlethaler, A. Qayyum, L. Viennot, "Optimized Link State Routing Protocol for Ad Hoc Networks," IEEE INMIC, Pakistan 2001.

[13] S.-J. Lee, W. Su, M. Gerla, "On-Demand Multicast Routing Protocol in Multihop Wireless Mobile Networks," ACM/Kluwer Mobile Networks and Applications, 2000.

[14] Thomas Kunz, Ed Cheng, "On-Demand Multicasting in Ad-Hoc Networks: Comparing AODV and ODMRP," Proc. 22nd IEEE International Conference on Distributed Computing Systems (ICDCS'02), pp. 453-454

[15] M. B. Pursley, J. M. Shea, "Nonuniform phase-shift-key modulation for multimedia multicast transmission in mobile wireless networks," IEEE Journal on Selected Areas in Communication, vol. 17, no. 5 pp. 774-783, May 1999.

[16] R. R. Choudhury, X. Yang, R. Ramanathan, N. Vaidya, "Using Directional Antennas for Medium Access Control in Ad Hoc Networks, ACM Intl. Conf. on Mobile Computing and Networking (MobiCom), September 2002.

[17] M. Takai, J. Martin, and R. Bagrodia, "Directional Virtual Carrier Sensing for Directional Antennas in Mobile Ad Hoc Networks," Proc. ACM Int. Symp.Mobile Ad Hoc Networking and Computing (MOBIHOC), Lausanne, Switzerland, Jun. 9-11 2002.

[18] R. R. Choudhury, N. Vaidya, "Impact of Directional Antennas on Ad Hoc Routing," Eighth International Conference on Personal Wireless Communication (PWC), Venice, Italy, September 2003.

[19] Jeffrey E. Wieselthier, Gam D. Nguyen, Anthony Ephremides, "Energy-Limited Wireless Networking with Directional Antennas: The Case of Session-Based Multicasting," Proc. of IEEE INFOCOM, New York, NY, June 2327, 2002

[20] A. Nasipuri, S. Ye, J. You, and R. E. Hiromoto, "A MAC Protocol for Mobile Ad Hoc Networks Using Directional Antennas," Proc. IEEE Wireless Communications and Networking Conference (WCNC), 2000.

[21] Z. Huang, C. Shen, C. Srisathapornphat, C. Jaikaeo, "Topology Control for Ad Hoc Networks with Directional Antennas," Proc. IEEE Int. Conference on Computer Communications and Networks, pp. 16-21, 2002.

[22] Ivan Stojmenovic, Mahtab Seddigh, Jovisa Zunic, "Dominating Sets and Neighbor Elimination-Based Broadcasting Algorithms in Wireless Networks," IEEE Trans. Parallel and Distributed Systems, Vol 13, No 1, 2002, pp. 14-25
[23] C. Perkins, P. Bhagwat, "Highly Dynamic DestinationSequenced Distance-Vector Routing (DSDV) for Mobile Computers," Proc. ACM SIGCOMM, Oct. 1994, pp 234244. 